\title{
DISEÑO E IMPLEMENTACIÓN DE UN PROTOTIPO PARA EL REGISTRO Y VERIFICACIÓN DE ACTIVOS FIJOS UTILIZANDO PLATAFORMA ANDROID Y TECNOLOGÍA NFC
}

\section{DESIGN AND IMPLEMENTATION OF A PROTOTYPE FOR REGISTRATION AND VERIFICATION OF FIXED ASSETS USING TECHNOLOGY NFC AND ANDROID PLATFORM}

\begin{abstract}
RESUMEN
Este documento plantea una propuesta de prototipo e implementación de una aplicación, construida en plataforma Android utilizando tecnologías NFC. La motivación para la elaboración de la aplicación es la problemática de la validación y control de flujo de activos fijos, bastante común en la actualidad. Con este desarrollo se busca proporcionar una solución a la tarea del registro y validación de entrada y salida de elementos físicos, que estarán relacionados con información almacenada en una base de datos donde se asociarán con una persona, en un lugar en específico. En el desarrollo de la aplicación se implementó una arquitectura de software basada en cliente-servidor contando con elementos tipo web y móvil, y acoplando diferentes tecnologías que convergen a un único servicio. Como trabajo futuro se destacara la posibilidad de extender la aplicación a cualquier organización que la requiera, también deben explorarse nuevas metodologías de seguridad en general para las etiquetas NFC que permitan generar una capa de seguridad eficiente y robusta. Finalmente, se obtiene como resultado una versión funcional del aplicativo probada en la Universidad Distrital Francisco José de Caldas de la ciudad de Bogotá en Colombia para el control de entrada y salida de dispositivos electrónicos, con resultados satisfactorios.
\end{abstract}

Palabras clave: Android, activos fijos, arquitectura de software, NFC, web service.

\begin{abstract}
This paper presents a proposal and implementation of a prototype application built on android platform using NFC technology, the motivation for development of the application is the problem of validation and control of equipment flow which is very common today. This development seeks a solution to the task of recording and validating input and output physical elements, which are listed in a database where they associate with a person in a specific place. In the development of the application we implemented a
\end{abstract}

\section{Alberto Acosta López}

Ingeniero de Sistemas, magister en Teleinformática y especialista en Gestión de Proyectos.

Universidad Distrital Francisco José de Caldas

aacostal@udistrital.edu.co

Bogotá, Colombia

Dago José Manzano González Estudiante de Ingeniería de Sistemas Universidad Distrital Francisco José de Caldas djmanznog@correo.udistrital.edu.co Bogotá, Colombia

\section{Carlos A. Martínez Morales}

Estudiante de Ingeniería de Sistemas Universidad Distrital Francisco José de Caldas

caralmartinezm@correo.udistrital.edu.co Bogotá, Colombia

Tipo: Artículo de investigación

Fecha de Recepción: Octubre 7 de 2013 Fecha de Aceptación: Diciembre 22 de 2013 
software architecture based on client-server type elements having web and mobile technologies into a unique service. Future work will emphasize the possibility of extending application to any organization that requires it. It must also explore new methodologies general security for NFC tags which can generate a layer of security more efficient and robust, it will consider a study market to assess generic costs involved in implementing this application in any company. Finally, a functional version of the application was tested in a university in the city of Bogotá in Colombia for controlling input and output of electronic devices.

Key words: Android, fixed assets, software architecture, NFC, web service.

\section{INTRODUCCIÓN}

En instituciones de masiva actividad y alto flujo de personas se requieren sistemas de control eficientes y seguros [1], los cuales proporcionen la suficiente robustez en cada uno de los escenarios de actividades recurrentes que se presentan [2], dentro de las cuales se encuentra el flujo de activos fijos. Algunas de las situaciones relacionadas con este aspecto de seguridad son las largas esperas en el proceso de verificación o validación de los activos, represamientos en el flujo de entrada y salida de personas en determinadas horas del día y la falta de optimización del tiempo de operación del personal encargado, todo asociado a la complicada tarea de implementación de un sistema de archivo para la gran cantidad de transacciones [3] o novedades que se presentan diariamente.

Actualmente está implementado en las diferentes entidades de nuestro entorno un sistema basado en el registro manual con el cual se lleva control y verificación del flujo de activos de las entidades. Este sistema almacena diariamente una cantidad elevada de eventos, generando problemas como los mencionados anteriormente, y entorpeciendo el buen funcionamiento de la labor diaria de control de activos fijos. Se precisa de métodos computacionales para optimizar y automatizar el proceso de verificación de activos fijos. En la tarea de registro y validación se han implementado algunas formas de validar objetos [4] las cuales son abordadas con tecnologías en medios electrónicos para el manejo de elementos cotidianos.

Gracias al considerable auge de desarrollos tecnológicos presentado en la actualidad [5], parte de los cuales se implementa en las ramas de la ingeniería y disciplinas afines, mayor cantidad de problemas cotidianos pueden abordase con desarrollos eficientes. Parte del desarrollo tecnológico tiene lugar en el campo de la ingeniería de software [6] dando lugar a aplicativos que se basan en diferentes tipos de plataforma entre las cuales tenemos los sistemas operativos para dispositivos móviles ampliamente extendidos y populares en la sociedad [7], como es el caso del sistema operativo Android, con tal versatilidad y portabilidad que podemos apreciar dispositivos simples como receptores de radiofrecuencia [8] de tecnología RFID, hasta elementos de última tecnología como los smart tv.

La tecnología NFC como sistema de transmisión de datos de campo cercano, que ofrece funcionalidades en los dispositivos móviles, es una ventaja actual ya que por medio de dispositivos electrónicos simples denominados etiquetas (Tags) podemos contar con un flujo de datos que ofrece conectividad entre dispositivos. Las aplicaciones de la tecnología NFC entran actualmente en muchos de los campos de operación como lo es el manejo del dinero 
electrónico, en la parte de publicidad (ya que se puede obtener información de vallas publicitarias inteligentes), en el servicio de transporte donde se ha implementado esta tecnología para acceder a estaciones y buses, servicios de negocios electrónicos entre teléfonos NFC.

El presente documento busca plantear un modelo arquitectural basado en tecnologías móviles y web, desarrollado en plataforma Android como solución al problema de registro, validación y flujo de activos fijos, utilizando comunicaciones NFC, y teniendo como soporte una base de datos.

\section{ANDROID Y ARQUITECTURA}

Android constituye un sistema operativo para dispositivos móviles. Las aplicaciones para Android se programan en el lenguaje Java y $\mathrm{C}++, \mathrm{y}$ son ejecutadas en una máquina virtual llamada Dalvik, el núcleo de la cual está basado este sistema operativo es Linux. Maneja la licencia de distribución apache 2.0 [9], lo que es denominado software de libre distribución. Android es patrocinado principalmente por la compañía Google y por un conjunto de empresas que conforman el Open Handset Alliance (OHA) [10].

Actualmente Android ha conseguido reunir los elementos necesarios para desarrollar y controlar las funcionalidades ofrecidas por los dispositivos móviles (GPS, NFC, Videojuegos, Etc.), estandarizando así el desarrollo de aplicaciones para una gran cuota del mercado actual. Además de todo ello, otro aspecto básico para entender la visión de Android es que proyecta facilitar la integración de estos dispositivos con las contingencias cada vez mayores ofrecidas por la Web. Por ejemplo, aplicaciones desarrolladas en Android que indican la posición en coordenadas a través del servicio Google Maps, introducir medios de pago electrónico por medio del chip NFC de un teléfono con Android, entre otras aplicaciones.

La arquitectura empleada de Android [11] se visualiza en cada una de las capas de la figura 1 , y se caracteriza porque cada una de las capas se apoya en los servicios ofrecidos en las capas inferiores.

La capa 1 corresponde al núcleo de Android, que utiliza a su vez el núcleo Linux como una capa de integración para el hardware disponible en los dispositivos móviles. Esta capa contiene los drives necesarios para que los componentes de hardware puedan comunicarse. La capa intermedia proporciona las librerías que son escritas en $\mathrm{C} / \mathrm{C}++$, que proporcionan la mayor parte de sus características. Los últimos niveles corresponden al framework de aplicaciones que está escrito directamente en lenguaje Java y que representa fundamentalmente la herramienta para el desarrollo de aplicaciones en Android.

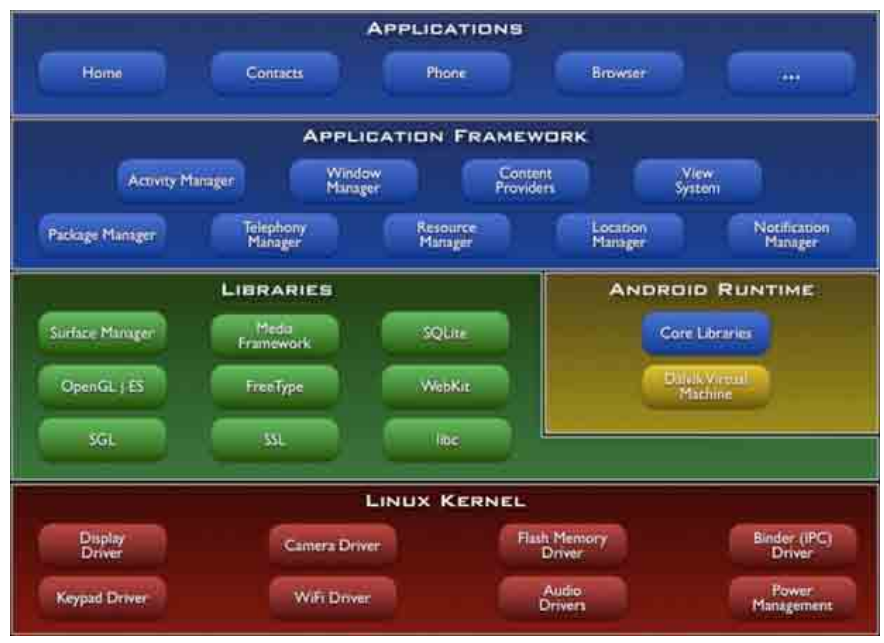

Figura 1. Arquitectura Android Fuente: [12] 


\subsection{Tecnología NFC}

Pensada en el uso de teléfonos móviles para el intercambio de información que permite identificar y validar el usuario. NFC fue desarrollada inicialmente por la compañía Sony en el año 2002. Esta tecnología consiste en la integración actual de dispositivos móviles, u otros dispositivos con la identificación por radiofrecuencia, que proporciona una comunicación entre artefactos electrónicos, usualmente trabaja a una distancia de 5 a $20 \mathrm{~cm}$ con una frecuencia de $13.56 \mathrm{MHz}$ y una taza de transferencia de 424 $\mathrm{Kb} / \mathrm{seg}$ [13], es compatible con etiquetas RFDI denominadas Tag, que pueden ser pasivas o activas. En el modo pasivo el dispositivo genera un campo de radiofrecuencia de corto alcance permitiendo que pueda leer y escribir datos en la memoria, en el modo activo ambos dispositivos generan su propio campo de radiofrecuencia para enviar los datos reconociéndose automáticamente.

La tecnología NFC actualmente da soporte a 4 tipos diferentes de etiquetas que varían en su capacidad de hasta $2 \mathrm{~Kb}$ y velocidades de hasta 212 Kbps; NFC es una tecnología de plataforma abierta estandarizada en la ISO/IEC 18092.

\section{DESARROLLO PROPUESTO}

El prototipo que se presenta en este estudio, cuyo fin de facilitar la labor de seguimiento de activos fijos se ha explicado previamente, pese a estar pensado para extenderse a cualquier tipo de institución que lo requiera, se llevará a cabo para prestar apoyo a la Universidad Distrital Francisco José de Caldas, en la ciudad de Bogotá, Colombia. El único motivo que justifica esta decisión es la necesidad de ejemplificar y probar su uso.

La metodología empleada para el desarrollo de esta implementación se basa en el desarrollo ágil a través del cual, de forma incremental e iterativa, se obtienen prototipos cuya completitud y correctitud aumenta en cada ciclo. En lugar de detallar de forma exahustiva el proceso mismo del desarrollo del software, en este documento se profundiza en sus caracteristicas, comenzando por su descripción general y revisión de su estructura. Posteriormente se muestra la estructura de datos (conforme a los requerimientos de datos necesarios para su operar y cumplimiento de su objetivo). En tercer lugar se ejemplifica su uso y funcionamiento en dispositivos móviles y en palataforma web, así como la comunicación de estas dos partes del desarrollo mediante un web service.

A continuación se detalla cada uno de los ítems que requirieron de especial atención para la elaboración del proyecto.

\subsection{Descripción general}

Como objetivo de la aplicación se busca facilitar el control de los activos utilizando un sitio web donde se encuentre la base de datos de los artículos registrados, así como también los propietarios y sus principales datos. Este aplicativo se comunicará a un aplicativo móvil a 7NFC ubicadas en los dispositivos registrados para completar sus operaciones y de consulta y escritura en la aplicación Web (ver figura 2).

El prototipo se realizó en base al flujo de activos que se produce en la universidad mencionada y es por este motivo que la estructura general del aplicativo se diseñó siguiendo sus requerimientos en específico: Guardar información del propietario de los dispositivos (estudiantes específicamente) que lo represente frente a la universidad, como un código estudiantil y un proyecto curricular.

En este sentido se tiene la información de los proyectos curriculares, así como información básica de los estudiantes que ingresan activos, esto incluye también la información que permite identificar los activos de forma única.

Se propone colocar una etiqueta de NFC para cada uno de los activos y de esta manera registrar el ID o el identificador único del chip para asociarlo con la información de los activos y sus propietarios. 


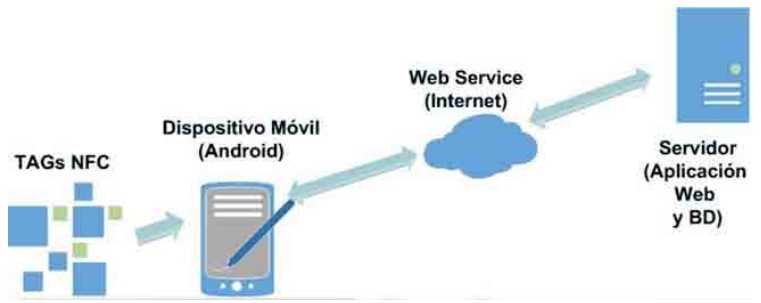

Figura 2. Estructura general del prototipo Fuente: elaboración propia

Para la selección de las etiquetas NFC a utilizar en el proyecto debe considerarse que existen algunas de carácter activo y otras que pasivas. Además, algunas etiquetas no se pueden ubicar sobre elementos metálicos ya que pierden su función. Algunas etiquetas permiten solamente lectura otras permiten escritura - lectura.

Para este prototipo se trabajó con etiquetas pasivas que permiten la lectura y la escritura, de preferencia aquellas que soportan utilizarse sobre elementos metálicos ya que, por ejemplo, se requiere para computadores que tengan cuerpo en aluminio.

Se propone colocar una etiqueta de NFC para cada uno de los activos y de esta manera registrar el ID o el identificador único del chip para asociarlo con la información de los activos y sus propietarios. Para este prototipo se trabajó con etiquetas pasivas (se activan únicamente cuando se tiene un dispositivo lector cercano) que permiten la lectura y la escritura de información, de preferencia aquellas que soportan utilizarse sobre elementos metálicos ya que, por ejemplo, se requiere para computadores que tengan cuerpo en aluminio.

\subsection{Estructura de datos}

Se ha planteado un modelo sencillo para la base de datos de tal forma que se pueda acceder fácilmente por medio de consultas a la información de un registro de equipos.

Se cuenta con las entidades mostradas en la figura 3.

Proyecto curricular: para evitar que se registren varias veces el mismo proyecto curricular escrito de diferentes formas.

Estudiante propietario: se busca hacer un registro de estudiante que cuente con algún elemento que desee registrar para ingreso almacenando la información básica de contacto a nivel de la universidad.

Activo: ya que un estudiante puede llegar a manejar varios dispositivos, se propone que se puedan asignar o registrar varios equipos para el mismo estudiante. Esto permitiría realizar un censo de dispositivos que se ingresan a la universidad y evitar anomalías.

Los registros se realizan de forma independiente y la misma base de datos se encarga de identificar cuál es el tipo de evento a registrar, así que el sistema está en la capacidad de identificar el ingreso o salida de dispositivos e incluso permitirá conocer en un momento dado la cantidad de aparatos ingresados por estudiantes presentes en la universidad.

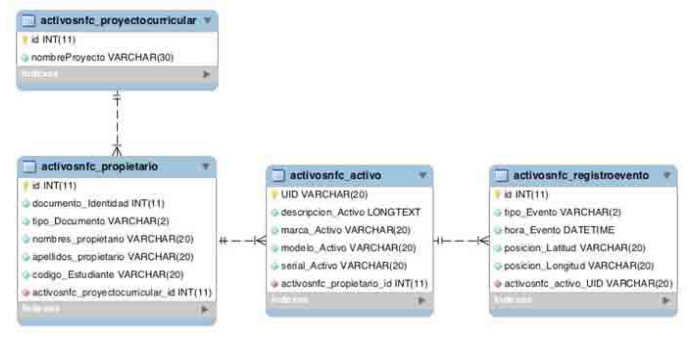

Figura 3. Modelo entidad relación Fuente: elaboración propia

\subsection{Aplicación móvil}

Una de las consideraciones para la aplicación móvil se refiere a la versión de Android a utilizar. En este caso la versión 4.0 o superior de Android para tener acceso a las funcionalidades del chip NFC. Las API de Android necesarias se encuentran disponibles desde dicha versión.

En general durante el desarrollo del aplicativo se determinó la importancia de organizar módulos que permitieran tener un acceso a los sensores y servicios web de forma que sea mucho más sencillo su mantenimiento y que se pueda agregar funcionalidades adicionales en 
caso de que sea necesario.

La aplicación móvil se organizó en los siguientes módulos:

NFC: Para tener una mejor organización en el aplicativo se planteó una clase de Java que diera soporte a las funciones básicas de acceso al chip NFC. En este caso se implementaron métodos para la lectura del chip el cual es la función más importante.

GPS: Para el acceso a información de posición se ha creado, al igual que para el caso de NFC, una clase que permite obtener los datos que se requieren para determinar la ubicación a partir del GPS del dispositivo.

JSON: Al igual que para el acceso a los sensores se decidió tener una clase encargada de la funcionalidad de conexión al Web service de tal manera que en caso de requerir conexión a más de una URL para realizar la conexión se pueda realizar sin necesidad de agregar más código o modificar el existente.

En la figura 4 se muestra una captura del aplicativo Web en funcionamiento, dividiéndose en tres partes principales: información geográfica, que señala la latitud y longitud del dispositivo móvil; la información de equipo, con los datos del activo; y la información del propietario, como un nombre y una identificación.

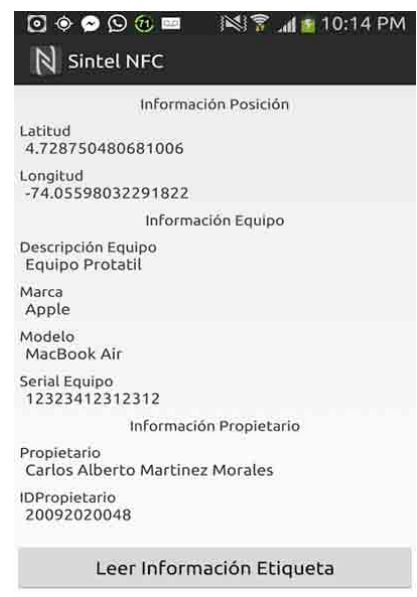

Figura 4. Aplicativo movil en funcionamiento Fuente: elaboración propia

\subsection{Aplicación Web}

La aplicación Web es el medio por el cual se ingresa la información de los activos y de los dueños de los mismos para que posteriormente sean consultados desde las entradas usando dispositivos móviles con la aplicación instalada en cada una de ellas.

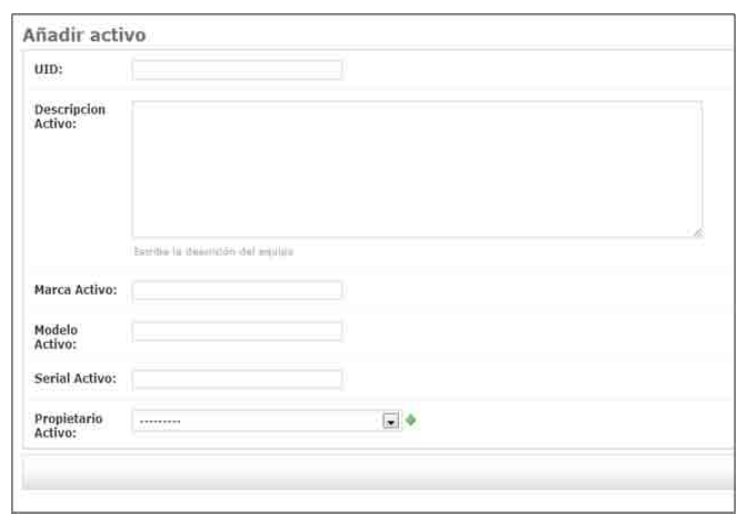

Figura 5a. Formulario de registro de la información Fuente: elaboración propia

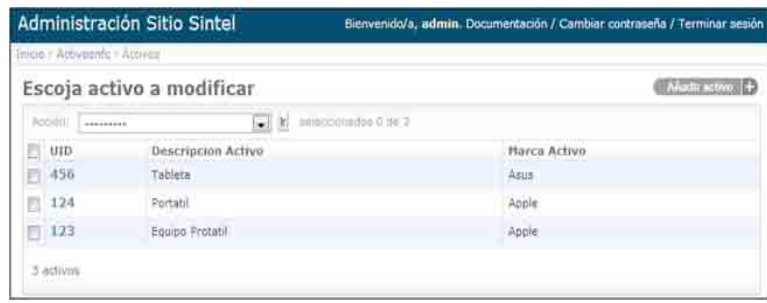

Figura 5b. Lista de activos registrados Fuente: elaboración propia

La aplicación Web se planteó sobre un framework Web de python llamado Django junto con un paquete adicional llamado piston que permite generar datos JSON desde el servidor y también permite recibir información por este medio desde el dispositivo móvil.

El resultado de la implementación de la funcionalidad principal del aplicativo Web, es decir, el registro de la información de los activos, se muestra en la figura 5. En la figura 5a se presenta un formulario para la recolección de la información que concierne a un activo, en la figura $5 \mathrm{~b}$ la lista de algunos activos de prueba registrados en el sitio Web. 


\subsection{Conexión}

Dado que las dos aplicaciones se encuentran en entornos diferentes se requiere el poder establecer un elemento común. En este caso de adopto el estándar JSON como lenguaje que permita comunicar la aplicación de Android con la aplicación Web que da soporte a toda la información. La aplicación móvil, con el código leído de la etiqueta NFC solicita la información pertinente del dispositivo a la aplicación Web (que contiene y controla la base de datos), frente a lo cual el aplicativo web responde con un archivo JSON como el que se observa en la figura 6 que contiene la información.

El resultado mostrado en la figura 5 es producto de la implementación real del servicio web desarrollado con ayuda del framework Django y consultado por el aplicativo android.

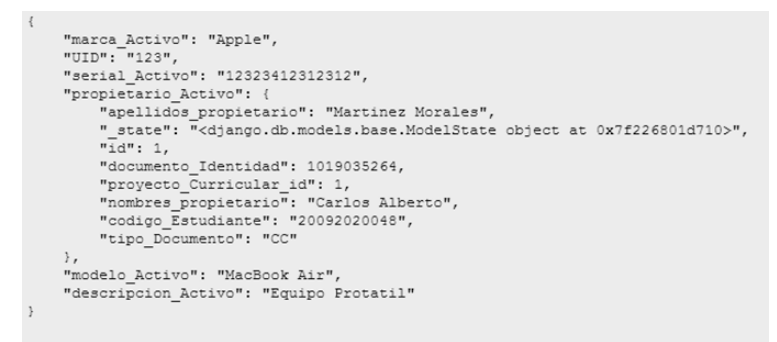

Figura 6. Documento JSON generado por el aplicativo Web

Fuente: elaboración propia

\section{TRABAJO FUTURO}

Después de llevar a cabo el proceso necesario para elaborar el prototipo descrito en el presente artículo, basado en las necesidades identificadas para el manejo de activos en la Universidad Distrital, y extensible a cualquier organización que requiera de apoyo en esta área de la seguridad, se hacen evidente ciertos tópicos de interés para la optimización y mayor aprovechamiento de este desarrollo.

En primer lugar debe mencionarse la necesidad de explorar nuevas metodologías de control y administración de seguridad para el acceso y lectura de las etiquetas NFC, más exhaustivas y adaptadas a cada entorno en particular. En el trabajo desarrollado se utiliza el identificador único propio de cada ETIQUETA que se guarda y se relaciona con un propietario en la base de datos, para luego acceder a la información almacenada en esta por parte de un usuario autorizado, sin embargo procedimientos asociados a la codificación de estos códigos y de las solicitudes de información realizadas a los Web services aportará sin duda características de gran valor.

Una mayor exploración en cuanto a las diferentes opciones que ofrece el mercado de la tecnología en términos de hardware para la detección y lectura de etiquetas NFC puede llevar a un manejo apropiado de costos que beneficie a la compañía que implemente el desarrollo, esto evitará que el valor de adquisición y mantenimiento se conviertan en una excusa para el mejoramiento del servicio de manejo de activos.

\section{CONCLUSIONES}

El sistema actual de control y registro de activos fijos utilizado en universidades y entidades en general difiere sustancialmente con el sistema propuesto en este artículo en dos principales aspectos: en primer lugar la metodología para la toma de datos de los activos, en el sistema actual consiste en un diligenciamiento manual de datos mientras en el sistema propuesto se realiza por un aplicativo web, este cambio permite guardar de forma segura y prolongada la información.

En segundo lugar, la toma de información de ingreso y egreso de los activos, tradicionalmente se realiza llenando un libro o minuta mientras en la propuesta presentada se espera que se realice con ayuda de la detección de la etiqueta mediante la aplicación web. Esta metodología propuesta permite un control inmediato de los activos registrados, así como una disminución en posibles errores en el llenado de información en los registros manuales (en datos como la hora o ubicación que se cargarían de forma transparente al usuario).

La aplicación de nuevas tecnologías como lo son las comunicaciones de campo cercano 
(NFC) trae consigo beneficios evidentes para el manejo operativo de ciertas áreas. La aplicación propuesta en este trabajo supone una notable mejoría a los manejos tradicionales de registros manuales para el flujo de activos.

Mediante información estadística generada por el desarrollo propuesto se puede llegar a facilitar la planeación estratégica de una compañía

\section{Referencias Bibliográficas}

[1] Lorino, Philippe. "El control de gestión estratégico." La gestión por actividades. Alfaomega Marcombo. México (1993) pp $1-5$.

[2] Fuentes, Andrea, Nathaly Rivera, and Raúl Pinos. Software Estadístico para Regresión. El caso de Regresión Logística y Regresión Poisson. Diss. ESPOL, 2012.

[3] Quintanilla R, Ernesto. "Jerarquía de centros según flujos de personas en el área metropolitana de Monterrey." Estudios Demográficos y Urbanos (1986), monterrey, Mexico pp 423-441.

[4] López, Maricela, and Graciela Cordero. "La experiencia de validar un instrumento para evaluar revistas académicas electrónicas en internet." Razón y palabra edición 31 Atizapán de Zaragoza, Mexico (2003) pp 1-2.

[5] Granollers i Saltiveri, Toni. "MPIu+ a. Una metodología que integra la Ingeniería del Software, la Interacción Persona-Ordenador y la Accesibilidad en el contexto de equipos de desarrollo multidisciplinares." (2004).

[6] Cuervo, Mauro Callejas. "La Ingeniería de Software Libre y sus herramientas aplicadas a proyectos informáticos." Reportes Técnicos en Ingeniería del en departamentos de seguridad y control de activos. De esta manera se pueden tomar directivas respecto a la cantidad de personal necesaria, dando espacio a estrategias dinámicas que varían según la demanda. De igual forma es posible llegar a identificar con una mayor eficiencia las necesidades tecnológicas para un servicio de calidad.

[7] Cheng, Yong-Hua, Wen-Kuang Kuo, and Szu-Lin Su. "An Android system design and implementation for telematics services." Intelligent Computing and Intelligent Systems (ICIS), 2010 IEEE International Conference on. Vol. 2. IEEE, 2010.

[8] Patauner, C., et al. "High Speed RFID/ NFC at the Frequency of $13.56 \mathrm{MHz}$." The First International EURASIP Workshop on RFID Technology, RFID. 2007 pp 5-9.

[9] Kew, Nick. Desarrollo de módulos $\mathrm{y}$ aplicaciones con Apache, ANAYA MULTIMEDIA 2008 pp 428-500.

[10] Alliance, Open Handset. "Open handset alliance." Retrieved August 26 (2011): 2011.

[11] Tomás Gironés, Jesús. "Arquitectura de Android." (2011) pp 11 - 100.

[12] Android Architecture. [En Linea]. Disponible en: http://elinux.org/Android Architecture [Consultado el 16 de Diciembre de 2013]

[13] Tapia, Dante I., et al. "Identificación por Radiofrecuencia: Fundamentos y Aplicaciones." Proceedings de las primeras Jornadas Científicas sobre RFID. Ciudad Real, Spain (2007) pp 1 -5. 